\title{
Unilateral Frontal Sinus Aspergillosis: A Case Report and Review Literature
}

\author{
Yannick Canton Kessely*, Felicien G. Toudjingar, Loobé Regis Mwabanyol, Mahamat Ali Bolti, \\ Aboubakar Aouami, Kader Ndiaye, Olivier Ouambi, Yusra Aboulbachar Ali, Momar Code Ba \\ Neurosurgery Department, Hopital “La Renaissance de Ndjari”, N’Djamena, Tchad \\ Email: *canton_kessely@yahoo.fr,toudji_felicien@yahoo.fr, looreg20@gmail.com, aaouami@gmail.com, kader_n@yahoo.fr, \\ boltiali@gmail.com,dr.ouambi.lo@yahoo.fr,dryusraaboulbachar@gmail.com,bamcode@hotmail.com
}

How to cite this paper: Kessely, Y.C., Toudjingar, F.G., Mwabanyol, L.R., Bolti, M.A., Aouami, A., Ndiaye, K., Ouambi, O., Ali, Y.A. and Ba, M.C. (2020) Unilateral Frontal Sinus Aspergillosis: A Case Report and Review Literature. World Journal of Neuroscience, 10, 101-106.

https://doi.org/10.4236/wjns.2020.102011

Received: March 5, 2020

Accepted: April 12, 2020

Published: April 15, 2020

Copyright $\odot 2020$ by author(s) and Scientific Research Publishing Inc. This work is licensed under the Creative Commons Attribution International License (CC BY 4.0).

http://creativecommons.org/licenses/by/4.0/

\begin{abstract}
Frontal sinus aspergillosis is a rare infection that can be usually associated with immunocompromised states and life-threatening with high mortality rate. The authors report in a developing country a case of immunocompetent patient with left unilateral frontal headache, associated with left eye ptosis. The Brain CT Scan revealed frontal sinus aspergillosis. She underwent surgery followed by itraconazole cure. The outcome is successful after a period of 14 months with resolution of symptomatology. Anatomopathological examination confirmed aspergillosis. They share their experience of management of this disease in a country where neurosurgery is very young specialty and where molecules against aspergillosis are not available.
\end{abstract}

\section{Keywords}

Aspergillosis, Fungal Infection, Sinus, Surgery

\section{Introduction}

Aspergillus is the most common fungal infection of the paranasal sinuses and usually appears as a chronic disease in an otherwise healthy patient. Frontal sinus aspergillosis is a rare infection that can be usually associated with immunocompromised states and life-threatening with high mortality rate [1]. The literature described a higher incidence of Aspergillus granuloma in Sudan, India, Pakistan and Saudi Arabia [2]. The frontal sinus is usually secondary to the participation of one of the other sinuses and it's an underdiagnosed disease in immunocompetent patients. We report a case of an immunocompetent woman, agricultural worker in Central Africa country: Chad, with a unilateral frontal sinus aspergilloma and a review of literature. 


\section{Case Report}

We report a case of a 52-year-old female patient seen in consultation for left unilateral frontal headache, associated with ptosis of the left eye for which she was previously followed in ophthalmology. The past medical history began 08 months ago with a unilateral headache partially calmed by over the counter analgesics. An ipsilateral ptosis gradually settled with exophthalmos. She had diplopia of the left eye. The patient did not have another known medical condition and was not on any immunosuppressive therapy. The cerebral CT scan revealed a hyperdense left intraorbital tissue formation with lysis of the roof of the orbit and the anterior wall of the blown fore sinus (Figure 1). The ipsilateral frontal sinus was filled and contained calcifications. The radiological diagnosis of aspergillosis was retained. There was no leukopenia biologically.

Surgical intervention was performed with a left unilateral frontal approach. There is a problem of greenish material from the frontal sinus with hard lumps (Figure 2). The frontal sinus was cranialized and separated from the anterior stage by the galea passed below the frontal lobe lining the anterior stage. The culture of the sample in search of aerobic or anaerobic germs was negative. After surgical intervention the ptosis disappeared. The pathology study concluded with aspergillus niger (Figure 3), and a treatment based on itraconazole was initiated. She was followed for a period of 14 months without anything in particular.

\section{Discussion}

Aspergillosis of the paranasal sinuses can arise from a hematogenous dissemination or a pulmonary infection or by direct contamination of the paranasal sinus. We did not find the starting point of the infection in our patient, but we think that it would probably have spread from latent pulmonary tuberculosis since the
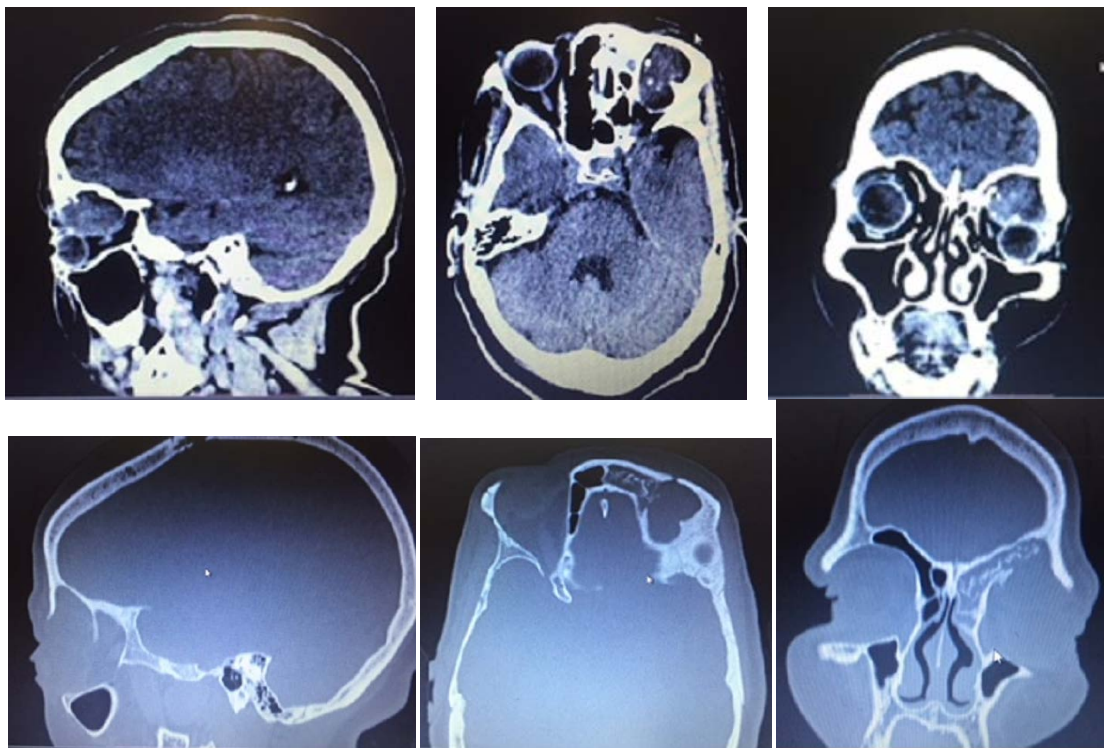

Figure 1. Brain CT scan showing filling of the left orbital cavity containing calcified material and a blown frontal sinus. 


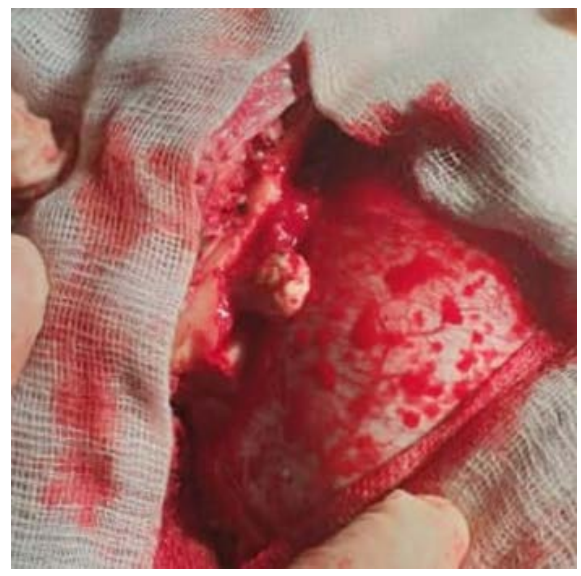

Figure 2. Outpouring of greenish material of the left frontal sinus.

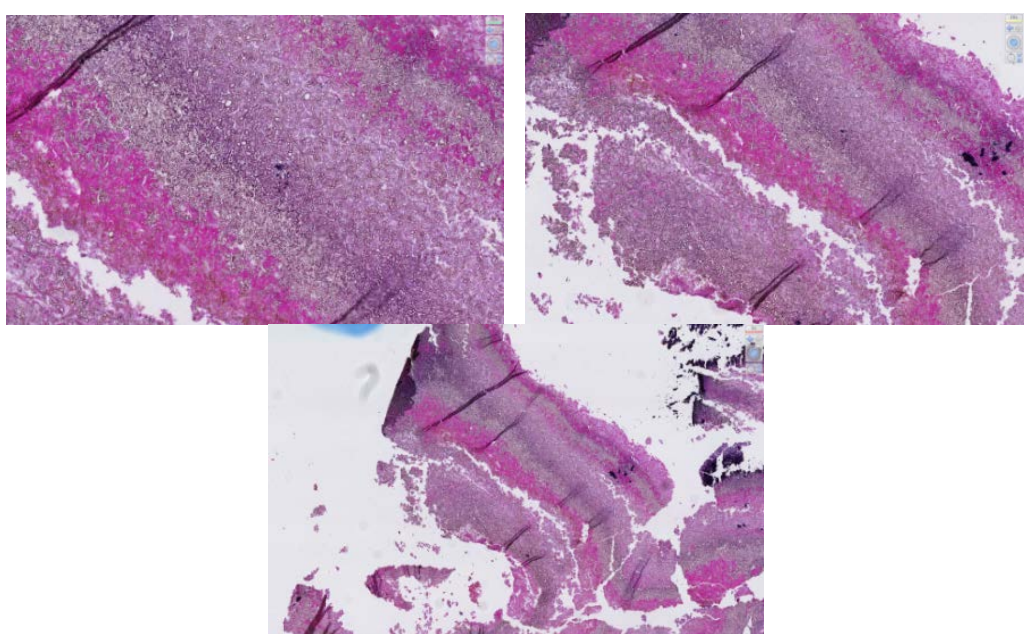

Figure 3. Spread of mycelial filaments generating compact, partially necrotic and calcified layers testifying "Aspergillus niger”.

patient is from an area where pulmonary tuberculosis can remain latent, which is associated with a tropical climate.

Aspergillosis disease has often been reported in areas that have a hot dry climate like in Sudan and the Middle East, and also in areas with temperate climates like India and Pakistan [2]. The patient lives in an area where precipitation reaches $1100 \mathrm{~mm}$ of rain per year with a temperate climate. This climate is similar to that of the South Sudan border. Humidity and a temperate climate, favor the growth of the fungus [2]. Dong Hoon reports in his study that aspergillus granulomas is more particularly to develop in immunocompromised individuals [3].

Leboime et al. reported that the risk of infection is increased in patients with immobility, skin breaks, joint surgery, rheumatoid arthritis, leukopenia, diabetes mellitus, and chronic lung diseases [4]. There is no evidence of pulmonary tuberculosis with the patient.

Different factors have been implicated in the onset of the disease such as, immunocompromised state, haemopoietic stem cell transplant, renal disease, solid 
organ transplant requiring the clinical use of immunosuppressive drugs, AIDS, sepsis, severe burns, uncontrolled diabetes mellitus, chemotherapy and radiotherapy in cancer therapy. The patient is a 52 year old female with a disease duration of about 9 months, with no evidence of immunosuppression (HIV and hepatitis B/C) chronic neutropenia, no otorhinolaryngological history and didn't receive any immunosuppressive treatments. For Maschmeyer et al. [5] an increase in population longevity, environmental pollution, alcoholism and other factors such as unhealthy levels of personal hygiene, sedentary lifestyles, obesity, invasive surgery with the use of prosthetic devices can contribute to immunocompromised situations.

Sinus aspergilloma may remain asymptomatic but the clinical manifestation of this rare disease is related to complications, by the orbital or intracranial invasion. Nasal symptoms are absent [6]. Our patient presented with an orbital extension of exophtalmos, ophthalmoplegia, visual compromise, and headaches evolving for 9 months. These symptoms have also been reported by Swoboda et al. [6], they reported the case of a patient with frontal sinus aspergilloma with a right sided pyocele expanding into the orbit. For intracranial aspergillosis, the patient presents with signs and symptoms of space occupying lesions [7], but all cases had a long history of headaches and nasal obstruction.

The culture of the sample taken in our patient was negative for the aerobic and anaerobic germs. This has also been reported by Pradeep et al. [7].

The entry of fungi into the host system is typically via the respiratory tract [3], via wounds for aspergillus conidia, via ingestion and/or via the hematogenous dissemination to the brain or sinuses. However, in our case study, we think that the entry was via the respiratory tract.

The frontal sinus is a rare location, according to Zachary et al. [8], fungal balls are common in middle aged or elderly women, often with a normal functioning immune system, and that workers in the agricultural fields or in the construction fields are more commonly exposed to inhale fungal spores [2]; which is the case of our patient; an agricultural worker.

The patient underwent a brain CT scan for an investigation. Some authors claim that CT scan is best for diagnosing sinus aspergilloma showing sinuses involved by the presence of a hyperdense mass with bony expansions or erosion of the cranial base [4]. Although a CT scan and an MRI can reveal the diagnosis. The definitive diagnostic investigation is the histopathologic confirmation.

According to the Siddiqui et al. [9] classification, our patient is a type 3 (Table $1)$.

Table 1. Siddiqui Classification.

\begin{tabular}{ccc}
\hline Type & Invasion & Mortality \\
\hline 1 & Intradurally & $66 \%$ \\
2 & Extradural intracranially & Intermediate outcome \\
3 & Limited to the orbital wall + cranial base & Excellent function \\
\hline
\end{tabular}


Infectious Diseases Society of America (IDSA) since 2016 [10] recommend both surgery and systemic Voriconazole as a first line therapy, or a lipid formulation of $\mathrm{AmB}$ reserved for those intolerant or refractory to Voriconazole. In our case, Voriconazole and Itraconazole are not available in our area, therefore Itraconazole was ordered from an outside country. The duration of therapy is not exactly defined, it varies from 3 to 12 months; it's sometimes related to the underlining disease. The duration of treatment by voriconazole varies among doctors. Preoperatively for decreasing mortality and morbidity [4], some doctors recommend liposomal Amphotericin B until the presence of Aspergillus is confirmed, and then Voriconazole is recommended [11]. However, we use Voriconazole without Amphotericin B after the diagnosis is confirmed. The surgical's goal is total removal of the fungi from the sinuse to avoid any reoccurrences.

For Gungor et al., despite surgery followed by oral Voriconazole, mortality rates varied from $28 \%$ - $85 \%$ [12], this fact can maybe be explained because of Voriconazole resistance. Otto and Delgaudio recommend following patients at least until there is a regeneration of the sinus mucosa. However in our case, our patient was followed for 14 months.

\section{Conclusion}

Frontal Aspergillus sinusitis is an opportunistic infection with a low incidence that can occur in immunocompromised patients. Early surgery and antifungal treatment can improve prognosis.

\section{Conflicts of Interest}

The authors declare no conflicts of interest regarding the publication of this paper.

\section{References}

[1] Bokhari, R., Baeesa, S., Al-Maghrabi, J. and Madani, T. (2014) Isolated Cerebral Aspergillosis in Immunocompetent Patients. World Neurosurgery, 82, e325-e333. https://doi.org/10.1016/j.wneu.2013.09.037

[2] Sundaram, C. and Murthy, J.M.K. (2011) Intracranial Aspergillus Granuloma. Pathology Research International, 2011, Article ID: 157320. https://doi.org/10.4061/2011/157320

[3] Lee, D.H., Yoon, T.M., Lee, J.K., Joo, Y.E., Park, K.H. and Lim, S.C. (2014) Invasive Fungal Sinusitis of the Sphenoid Sinus. Clinical and Experimental Otorhinolaryngology, 7, 181-187. https://doi.org/10.3342/ceo.2014.7.3.181

[4] Leboime, A., Berthelot, J.-M., Allanore, Y., Khalil-Kallouche, L., Herman, P. and Lioté, P.O.F. (2009) Sinus aspergilloma in Rheumatoid Arthritis before or during Tumor Necrosis Factor-Alpha Antagonist Therapy. Arthritis Research \& Therapy, 11, No. 6. https://doi.org/10.1186/ar2849

[5] Maschmeyer, G., Beinert, T. and Buchheidt, D. (2009) Diagnosis and Antimicrobial Therapy of Lung Infiltrates in Febrile Neutropenic Patients: Guidelines of the Infectious Diseases Working Party of the German Society of Haematology and Oncology. European Journal of Cancer, 45, 2462-2472. 
https://doi.org/10.1016/j.ejca.2009.05.001

[6] Swoboda, H.R. and Ullrich, R. (1992) Aspergilloma in the Frontal Sinus Expanding into the Orbit. Journal of Clinical Pathology, 45, 629-630.

https://doi.org/10.1136/jcp.45.7.629

[7] Sharma, P., Bhaisora, K.S., Pandey, S., Srivastava, A.K., Pani, K.C., Sardhara, J., Das, K.K., Mehrotra, A., Sahu, R.N., Jaiswal, A.K. and Behari, S. (2018) Aspergilloma Mimicking Olfactory Groove Meningioma. Asian Journal of Neurosurgery, 13, 436-439. https://doi.org/10.4103/1793-5482.228571

[8] Soler, Z.M. and Schlosser, R.J. (2012) The Role of Fungi in Diseases of the Nose and Sinuses. American Journal of Rhinology \& Allergy, 26, 351-358.

https://doi.org/10.2500/ajra.2012.26.3807

[9] Siddiqui, A., Shah, A.A. and Bashir, S.H. (2004) Craniocerebral Aspergillosis of Sinonasal Origin in Immunocompetent Patients: Clinical Spectrum and Outcome in 25 Cases. Neurosurgery, 55, 602-613. https://doi.org/10.1227/01.NEU.0000134597.94269.48

[10] Patterson, T.F., Thompson, G.R., Denning, D.W., Fishman, J.A., Hadley, S., Herbrecht, R., Kontoyiannis, D.P., Marr, K.A., Morrison, V.A., Nguyen, M.H., Segal, B.H., Steinbach, W.J., Stevens, D.A., Walsh, T.J., Wingard, J.R., Young, J.A. and Bennett, J.E. (2016) Practice Guidelines for the Diagnosis and Management of Aspergillosis: Update by the Infectious Diseases Society of America. Clinical Infectious Diseases, 63, 433-442. https://doi.org/10.1093/cid/ciw444

[11] Walsh, T.J., Anaissie, E.J. and Denning, D.W. (2008) Treatment of Aspergillosis: Clinical Practice Guidelines of the Infectious Diseases Society of America. Clinical Infectious Diseases, 46, 327-360. https://doi.org/10.1086/525258

[12] Gungor, A., Adusumilli, V. and Corey, J.P. (1998) Fungal Sinusitis: Progression of Disease in Immunosuppression-A Case Report. Ear, Nose \& Throat Journal, 77, 207-210. https://doi.org/10.1177/014556139807700311 